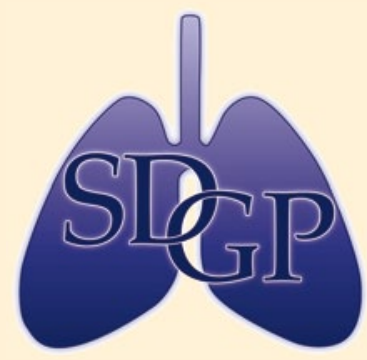

Pneumologe 2011 · 8:128-128 DOI 10.1007/s10405-011-0490-x ○ Springer-Verlag 2011
Vorstand der SDGP

Vorsitzender

Prof. Dr. med. Felix J. F. Herth

Thoraxklinik Heidelberg

felix.herth@thoraxklinik-heidelberg.de

Stellvertretender Vorsitzender

Prof. Dr. med. Joachim H. Ficker

Klinikum Nürnberg Nord

joachim.ficker@klinikum-nuernberg.de

Geschäftsführer

Dr. med. Johannes Schildge

St. Vincentius Kliniken Karlsruhe

johannes.schildge@vincentius-ka.de

\title{
25. Fortbildungstage der Süddeutschen Gesellschaft für Pneumologie
}

\author{
Datum: 07.-08.10.2011 \\ Ort: Liederhalle Stuttgart \\ Tagungspräsident: PD Dr. med. Martin Hetzel, \\ Chefarzt, Klinik für Pneumologie, Beatmungsmedi- \\ zin und Allgemeine Innere Medizin, Krankenhaus \\ vom Roten Kreuz, 70372 Stuttgart
}

\section{Zielgruppen \\ - Ärzte \\ - Assistenz- und Pflegepersonal \\ - Patienten}

\section{Schwerpunktthemen}

- Asthma

- COPD

- Lungentumorerkrankungen

- Tabakentwöhnung

- Atmungsstörungen im Schlaf

- Allergie

\section{Kurse}

- Bronchoskopiekurs

- Lungenfunktionskurs

- Bildgebung in der Pneumologie

\section{Grußwort des Tagungs- präsidenten}

Sehr geehrte Kolleginnen und Kollegen,

zur 25. Fortbildungstagung der Süddeutschen Gesellschaft für Pneumologie lade ich Sie als Tagungspräsident herzlich in die Landeshauptstadt Stuttgart ein. Der Kongressort ist das Kultur- und Kongresszentrum Liederhalle, mitten im Herzen von Stuttgart.

Die 25. Fortbildungstage der Süddeutschen Gesellschaft für Pneumologie werden keine wissenschaftliche Veranstaltung sein.

Es erwarten Sie keine Diskussionen zur statistischen Signifikanz von Studienergebnissen oder zum wissenschaftlichen Evidenzgrad von Konsesusempfehlungen.

Das Hauptprogramm ist als Fortbildungsveranstaltung für Allgemeinärzte und hausärztlich tätige Internisten konzipiert. Erfahrene, am Patienten tätige Pneumologen werden mit Praxisbezug zu Themen der Pneumologie, wie Asthma, COPD, Lungentumorerkrankungen, Tabakentwöhnung, Atmungsstörungen im Schlaf und Allergie referieren.

Parallel dazu bieten die Fortbildungstage für Ärzte in der Weiterbildung zum Internisten oder zum Pneumologen Weiterbildungsmöglichkeiten zu spezialisierten pneumologischen Themen:

Es werden Bronchoskopiekurse, Lungenfunktionskurse, Kurse zum thorakalen Ultraschall und zur Bildgebung in der Pneumologie angeboten.

Die 25. Fortbildungstage leisten aber mehr als die Auffrischung gewonnenen Wissens oder die Vermittlung von neuen Erkenntnissen. Das Gespräch untereinander, der Erfahrungsaustausch und die begleitende Diskussion mit Kollegen werden dazu beitragen, dass neue Ideen und pragmatisches Vorgehen mit in den beruflichen Alltag genommen werden können.

Mein Team und ich freuen uns darauf, Sie im Herzen Stuttgarts zu einer praxisrelevanten Fortbildung begrüßen zu dürfen!

Mit herzlichen Grüßen Ihr

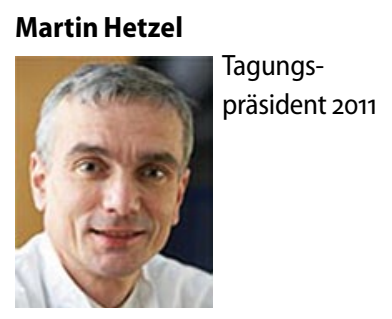

Wir bringen Ihnen die Pneumologie ins Haus 